\title{
IDENTIFICACIÓN DE PARÁMETROS DE COLECTORES SOLARES PLANOS MEDIANTE ALGORITMOS GENÉTICOS
}

\author{
Manuel G. Satué, Manuel G. Ortega, Francisco R. Rubio \\ Departamento de Ingeniería de Sistemas y Automática, Universidad de Sevilla \\ \{mgarrido16, mortega, rubio\}@us.es
}

\begin{abstract}
Resumen
En este trabajo se identifican los parámetros de un modelo de parámetros distribuidos de colectores solares planos mediante un algoritmo genético de codificación real utilizando datos de una planta piloto experimental.
\end{abstract}

Palabras Clave: Modelado de plantas solares, Colectores solares planos, Ajuste de parámetros, Algoritmo genético de codificación real.

\section{INTRODUCCIÓN}

El objetivo de las plantas solares térmicas es transferir el calor procedente de la radiación solar a un fluido para, en una segunda etapa, transformar la energía en forma de calor a otra forma de energía. Normalmente las plantas solares producen electricidad mediante una turbina y un alternador, pero también es posible utilizar la energía del fluido para producir frío mediante un ciclo termodinámico en sistemas de aire acondicionado.

El Sol es una fuente inagotable de energía, pero es una energía de baja densidad de potencia que presenta una serie de inconvenientes desde el punto de vista del control automático: su naturaleza es intermitente, la aparición esporádica de nubes hace que las variaciones en el nivel de radiación solar afecten rápidamente a la temperatura de salida del fluido, y además la radiación no es constante durante el día. Por este motivo interesa disponer de un modelo del campo de colectores solares, ya sea para su utilización offline para el ajuste de controladores o para su utilización online como predictor.

Este artículo se centra en las plantas solares de colectores planos para producción de frío, en las cuales el fluido de trabajo es agua en estado líquido. A nivel constructivo, un colector solar plano se compone de una o más cubiertas de vidrio, una placa absorbente metálica en contacto con los tubos por los que circula el agua y por último, una capa de material aislante como se muestra en la figura 1. La función de la cubierta es proteger la placa absorbente y evitar la pérdida de calor. La placa absorbente es de color negro para maximizar la eficiencia en la captación de calor. El aislante reduce las pérdidas de calor por la base del colector.

Los modelos dinámicos de colectores se pueden clasificar [9] en:

- Modelos de capacidad única (un nodo)

- Modelos de parámetros distribuidos en la dirección del flujo ( $1 \times \mathrm{N}$ nodos $)$

- Modelos de parámetros distribuidos en la dirección del flujo y en la dirección transversal al flujo ( $\mathrm{M} \times \mathrm{N}$ nodos)

Algunos autores $[2,9,11]$ han desarrollado modelos complejos que tienen en cuenta un número elevado de parámetros relacionados con los fenómenos de transferencia de calor que se producen entre los distintos elementos implicados (convección natural entre la cubierta y el aire interior, radiación entre la cubierta y la placa absorbente, conducción entre la placa absorbente y el aislante, etc.) así como la geometría interna del panel, etc. La cuestión es que los fabricantes no suelen proporcionar información de las propiedades de los materiales que emplean ni tampoco de la geometría interna, haciendo que ajustar un modelo complejo sea una tarea difícil. En el campo del control automático es conveniente que el modelo contemple las dinámicas más representativas del proceso usando el menor número de parámetros posible. En el presente artículo se ha utilizado un modelo de parámetros distribuidos en la dirección del flujo que aglutina todas las capacidades de los distintos materiales en una única capacidad térmica de todo el colector [8].

Para ajustar los parámetros del modelo se ha utilizado un algoritmo genético (AG) de codificación real alimentado con datos experimentales de una planta piloto experimental de la Escuela Técnica Superior de Ingeniería de la Universidad de Sevilla. Los algoritmos genéticos son métodos para la resolución de problemas de optimización que basan su funcionamiento en la teoría de la evolución por selección natural. Concretamente, hacen uso de los conceptos de selección, mutación, clonación y cruce de individuos de una población para evolucionar hacia el óptimo de una función objetivo. La principal 
diferencia entre los algoritmos genéticos y los métodos clásicos basados en gradiente es que en los primeros, la exploración el espacio tiene un fuerte componente estocástico mientras que en los segundos es determinista. Una de las ventajas de los AG es que permiten resolver problemas que no están bien acondicionados para los métodos clásicos, por ejemplo cuando la función objetivo no es derivable o está afectada por ruido. Otra ventaja es que tienen menos probabilidades de estancarse en mínimos locales, ya que exploran el espacio en múltiples puntos a la vez. Como contrapartida, los algoritmos genéticos no garantizan que se encuentre la solución óptima (exacta) del problema.

El resto del artículo está estructurado de la siguiente manera: en la Sección 2 se describe la planta experimental, en la Sección 3 se describe el modelo matemático de colector solar plano utilizado, en la Sección 4 se explica la metodología utilizada para el ajuste de parámetros, se dan algunos detalles sobre algoritmos genéticos y se presenta el pseudocódigo del AG utilizado, y finalmente, en la Sección 5 se presentan resultados experimentales.
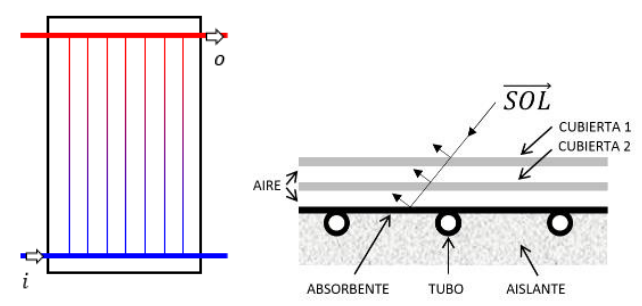

Figura 1: Detalles constructivos de un colector solar plano. Izq) vista frontal. Der) sección transversal.

\section{PLANTA SOLAR}

La instalación de refrigeración solar de la Escuela Técnica de Ingenieros de Sevilla consta de un equipo de absorción con una potencia frigorífica nominal de $35 \mathrm{~kW}$, un sistema de aporte de energía térmica y un sistema de evacuación de calor. La fuente de calor está compuesta por un conjunto de captadores solares térmicos que transforman la energía incidente en forma de radiación en energía interna del fluido caloportador (agua). En condiciones nominales, la energía térmica suministrada por el sistema de captación es de $50 \mathrm{~kW}$. Como complemento al sistema de captadores existe un sistema de energía auxiliar, una caldera de gas natural de $60 \mathrm{~kW}$, y dos tanques de almacenamiento de agua caliente que tienen la función de aportar energía cuando la radiación solar sea insuficiente. La energía que disipa el sistema de refrigeración por absorción es evacuada en una torre de refrigeración de $80 \mathrm{~kW}$. La demanda de energía térmica puede provenir de dos fuentes: la instalación de climatización de la segunda planta del edificio de laboratorios (carga real) o un sistema simulador de cargas consistente en una bomba de calor y un intercambiador agua-agua.

El sistema de captación tiene una superficie útil de $153.36 \mathrm{~m}^{2}$ y está compuesto por cuatro campos de captadores solares MADE modelo 4000E. Cada uno de los campos está compuesto por 18 captadores de $2.13 \mathrm{~m}^{2}$ cada uno (38.34 $\mathrm{m}^{2}$ en total), dispuestos en tres baterías de seis colectores, como se observa en la figura 2. Los cuatro campos (CC1, CC2, CC3 y CC4) están conectados en paralelo entre sí. Los colectores tienen orientación Sur y están inclinados $30^{\circ}$ respecto de la horizontal.

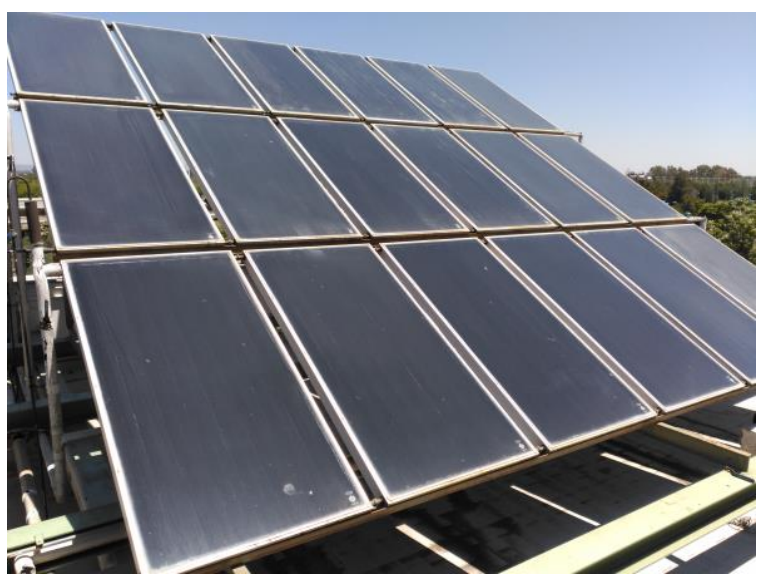

Figura 2: Campo de colectores CC4.

El sistema se controla con un PLC Allen Bradley CompactLogix L33ER que actúa sobre las electroválvulas, válvulas de tres vías, bombas $\mathrm{y}$ máquinas, y toma medidas de temperaturas, caudales y presiones de los diferentes circuitos, además de las medidas de una estación meteorológica. La supervisión se realiza mediante un cliente HMI conectado al servidor OPC RSLinx del fabricante Allen Bradley.

\section{MODELADO DE UN COLECTOR}

Como se dice en la introducción, se ha utilizado un modelo de parámetros distribuidos en la dirección del flujo que aglutina todas las capacidades de los distintos materiales en una única capacidad térmica de todo el colector.

Si se aplica un balance de energía a un elemento diferencial de fluido en la dirección del flujo se obtiene la siguiente ecuación en derivadas parciales:

$$
W C_{T} \frac{\partial T_{F}}{\partial t}=F^{\prime} W\left(I_{G}-U_{L}\left(T_{F}-T_{a m b}\right)\right)-\dot{m} C_{p} \frac{\partial T_{F}}{\partial x}
$$


donde el significado de las variables y parámetros se explica en la tabla 1.

Este modelo presenta las limitaciones que se enumeran a continuación:

-El modelo auna todas las capacidades de los distintos materiales en una única capacidad de todo el colector.

-No se contempla el efecto del viento (velocidad y dirección) en las pérdidas de calor.

Aparte, el calor específico del agua y su densidad se consideran constantes. Aunque estas propiedades dependen de la temperatura y la presión, en el rango de operación de un colector solar su variación es muy pequeña.

Tabla 1: Nomenclatura 1

\begin{tabular}{|c|l|}
\hline Nombre & Descripción \\
\hline$T_{F}$ & Temperatura del fluido $\left({ }^{\circ} \mathrm{C}\right)$ \\
$W$ & Ancho del colector $\left(\mathrm{m}^{2}\right)$ \\
$F^{\prime}$ & Factor de eficiencia del colector \\
$U_{L}$ & Coeficiente global de pérdidas al \\
& ambiente $\left(\mathrm{W} / \mathrm{m}^{2}{ }^{\circ} \mathrm{C}\right)$ \\
$T_{a m b}$ & Temperatura ambiente $\left({ }^{\circ} \mathrm{C}\right)$ \\
$\dot{m}$ & Caudal másico de fluido $(\mathrm{Kg} / \mathrm{s})$ \\
$Q$ & Caudal volumétrico de fluido $(\mathrm{l} / \mathrm{h})$ \\
$C_{p}$ & Calor específico del fluido $\left(\mathrm{J} / \mathrm{Kg}{ }^{\circ} \mathrm{C}\right)$ \\
$I_{G}$ & Irradiación global en el plano del \\
& colector $\left(W / \mathrm{m}^{2}\right)$ \\
$C_{T}$ & Capacidad térmica por unidad de área \\
& del colector $\left(\mathrm{J} / \mathrm{m}^{2}{ }^{\circ} \mathrm{C}\right)$ \\
\hline
\end{tabular}

\subsection{MODELO DE PARÁMETROS CONCENTRADOS}

Integrando la ecuación (1) a lo largo de la dimensión $x$, obtenemos que la variación de energía interna del colector se escribe de la siguiente manera:

$C_{T} \frac{\partial T_{F}}{\partial t}=F^{\prime}\left(I_{G}-U_{L}\left(T_{F}-T_{a m b}\right)\right)-\dot{m} C_{p}\left(T_{S}-T_{E}\right)$

Donde $T_{S}$ y $T_{E}$ son las temperaturas de salida y entrada del fluido al colector, y $T_{F}$ es la temperatura media del fluido. Para resolver la ecuación (2) es necesario relacionar la temperatura media con la temperatura de salida:

$$
T_{F}=\left(T_{S}+T_{E}\right) / 2
$$

La ecuación (2) se denomina modelo de capacidad simple (un único nodo de capacidad).

\subsection{MODELO DE DISTRIBUIDOS}

Para resolver la ecuación (1) se discretiza el espacio (la longitud del colector, figura 3) en $\mathrm{N}$ nodos obteniendo así un modelo consistente en $\mathrm{N}$ ecuaciones diferenciales, cada una asociada a un nodo $j, j=$ $1 \ldots N$.

$$
\begin{gathered}
W C_{T} \frac{\partial T_{F_{j}}}{\partial t}=F^{\prime} W\left(I_{G}-U_{L}\left(T_{F}-T_{a m b}\right)\right)-\dot{m} C_{p} \frac{\Delta T_{F_{j}}}{\Delta x} \\
\frac{\partial T_{F_{j}}}{\partial x} \approx \frac{\Delta T_{F_{j}}}{\Delta x}=\frac{T_{F_{j}}-T_{F_{j-1}}}{\Delta x}
\end{gathered}
$$
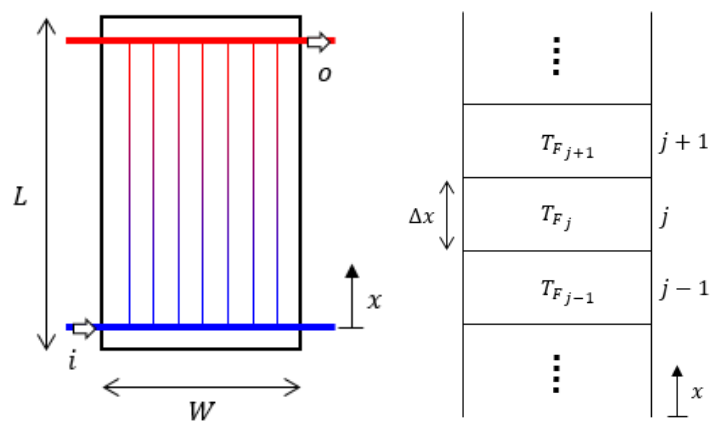

Figura 3: Discretización espacial de un colector en la dirección del flujo.

El sistema de ecuaciones se resuelve integrando numéricamente con un método RK4 (Runge-Kutta 4). La figura 4 muestra un resultado de simulación de un colector en el que se impone como condición inicial una distribución de temperatura uniforme e igual a la temperatura de entrada al colector, $T E C$, y la condición de contorno $\left(T_{F_{1}}=T E C\right)$ se mantiene constante durante todo el tiempo de la simulación. El número de nodos óptimo se ha establecido en 32 . La tabla 2 muestra la nomenclatura utilizada.

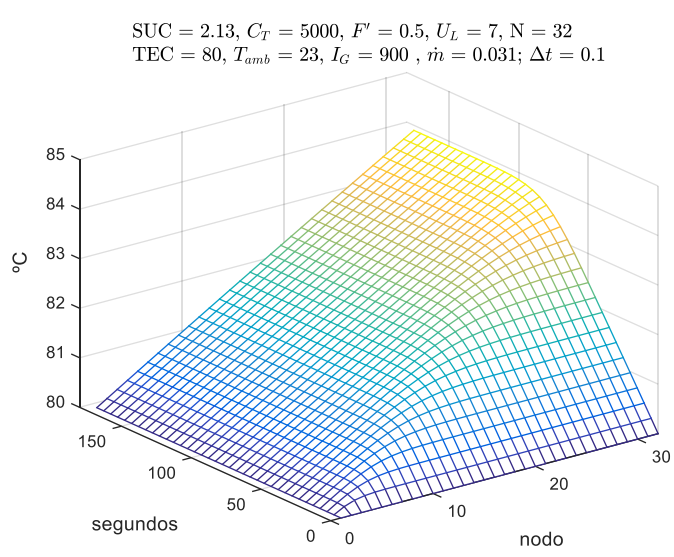

Figura 4: Evolución de la distribución de la temperatura a lo largo del colector con el tiempo. 
Tabla 2: Nomenclatura 2

\begin{tabular}{|c|l|}
\hline Nombre & Descripción \\
\hline$S U C$ & Superficie útil de captación $\left(\mathrm{m}^{2}\right)$ \\
$L$ & Longitud del colector $\left(\mathrm{m}^{2}\right)$ \\
$T E C$ & Temperatura entrada a colector $\left({ }^{\circ} \mathrm{C}\right)$ \\
$T S C$ & Temperatura salida de colector $\left({ }^{\circ} \mathrm{C}\right)$ \\
$N$ & Número de nodos \\
$\Delta t$ & Paso de integración $(s)$ \\
\hline
\end{tabular}

\subsection{MODELADO DE UN CAMPO DE COLECTORES}

Para modelar un campo de colectores a partir del modelo de un colector, dado que los colectores están conectados en paralelo y solo se dispone de una medida del caudal que circula por todos los colectores del campo, se ha considerado que un campo de colectores es equivalente a un colector de mayor supercicie de captación por el cual circula un caudal de agua mayor (figuras 5 y 6 ).

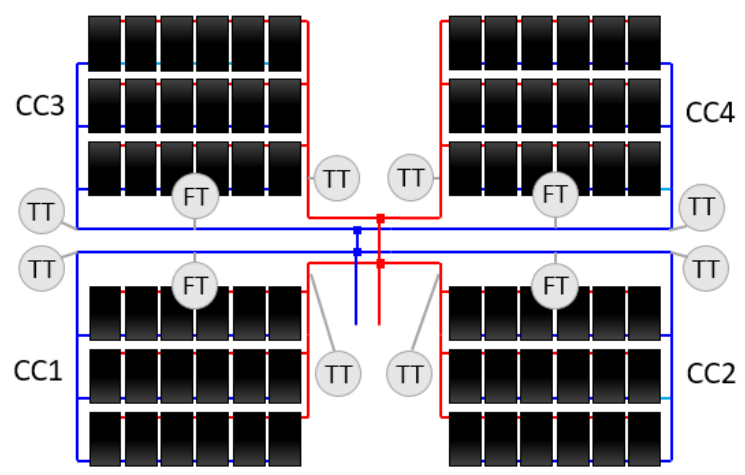

Figura 5: Disposición y conexionado de los campos de colectores y localización de los sensores de temperatura (TT) y caudal (FT).

Esta analogía implica la simplificación de considerar que por las tres filas de colectores circula el mismo caudal, cosa que no ocurre en la realidad. Debido a que cada fila de colectores se encuentra a diferente altura, la gravedad hace que el caudal se distribuya de forma diferente por las tres ramas (ménor caudal en la fila más alta y mayor caudal en la más baja).

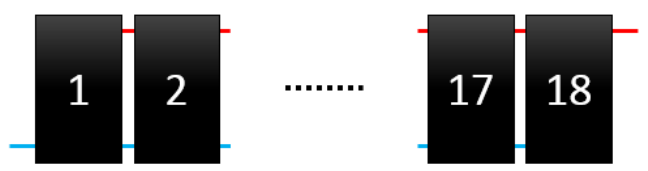

Figura 6: Simplificación de la distribución de los paneles de un campo de colectores.

Como se puede observar en la figura 5, se dispone de medidas de temperatura (TT, sondas PT100) a la entrada y la salida de cada campo así como del caudal volumétrico (FT) que circula por cada campo de colectores. También se dispone de una estación meteorológica que proporciona medidas de irradiancia, temperatura ambiente y velocidad $y$ dirección del viento.

\section{MÉTODO DE AJUSTE DE PARÁMETROS}

Para el ajuste del modelo se ha elegido un algoritmo genético por los motivos señalados en la introducción.

Se pretende obtener los valores de los parámetros desconocidos del modelo: la eficiencia del colector $F^{\prime}$, el coeficiente global de pérdidas al ambiente $U_{L}$, y la capacidad térmica del colector por unidad de área $C_{T}$. Hay que hacer notar que estos tres parámetros pueden variar en el tiempo por el propio envejecimiento de la instalación y por la suciedad que se acumula en la cubierta de los colectores (la eficiencia varía en función del grado de limpieza del colector). Hay que matizar que, si bien los valores exactos de los tres parámetros son desconocidos, sus valores aproximados sí son estimables. Esta información se utilizará para restringir el espacio de búsqueda del algoritmo genético.

Las entradas del algoritmo genético son las trayectorias obtenidas de un ensayo real de la planta. Se almacenan las trayectorias de TEC, TSC, $\dot{m}, I_{G}$ y $T_{a m b}$ muestreadas cada cinco segundos (ver tabla $1 \mathrm{y}$ tabla 2).

Los ensayos realizados consisten en dar escalones al caudal mediante una bomba con variador de frecuencia, e introducir perturbaciones en la temperatura de entrada a colectores con el simulador de carga (bomba de calor e intercambiador). Los ensayos también contemplan perturbaciones en la radiación solar producidas por las nubes.

Como se explica en el siguiente apartado, el AG necesita que se le proporcione un índice de ajuste o función objetivo. El índice de ajuste elegido es la integral de error cuadrático, ISE:

$$
\begin{gathered}
I S E=\int_{o}^{T} e(t)^{2} d t \\
e(t)=T S C_{\text {real }}(t)-T S C_{\text {simulada }}(t)
\end{gathered}
$$

\subsection{DETALLES DEL ALGORITMO GENÉTICO}

Los algoritmos genéticos trabajan con una población de individuos. Un individuo consiste en un vector de variables reales de dimensión igual al número de variables de entrada del problema, de manera que cada individuo representa una solución factible del 
problema de optimización (normalmente sub-óptima). El vector que constituye un individuo suele denominarse genoma y sus componentes, genes. Cada individuo tendrá asociado por tanto un valor resultante de la evaluación de la función objetivo que se denomina mérito.

En cada iteración del algoritmo, para crear una nueva población se seleccionan ciertos individuos de la generación actual, los padres, y se cruzan para dar lugar a nuevos individuos, los hijos. En un problema de maximización, cuanto mayor sea el mérito de un individuo, mayor será la probabilidad de que sea seleccionado para cruzarse con otro individuo. Para tratar un problema de minimización lo más sencillo es transformarlo en un problema de maximización:

$$
\min f(x) \equiv \max -f(x)
$$

El resultado del cruce de dos individuos es otra pareja de individuos con sus características mezcladas. Para mantener el tamaño de la población en las sucesivas iteraciones del algoritmo se cruzan la mitad de los individuos. Normalmente también se clona al menos el mejor individuo de la generación anterior. A esto se le suele llamar elitismo.

El último paso para completar una nueva generación consiste en aplicar mutaciones a un porcentaje de la población. La mutación consiste en introducir cambios aleatorios en los genes de un individuo.

\subsubsection{Mecanismo de selección}

La selección de individuos de una población para generar la siguiente generación se basa en el mérito de los individuos. Los individuos con mayor mérito tendrán más posibilidades de ser seleccionados. Por tanto, hay que asignar probabilidades a los individuos basadas en su mérito. Existen diferentes maneras de calcular estas probabilidades [6]. Una de las más conocidas es la selección proporcional:

$$
\operatorname{Prob}{ }^{p r o p}\left(P^{i}\right)=\frac{f\left(P^{i}\right)}{\sum_{j=1}^{N} f\left(P^{j}\right)}
$$

Donde $P^{i}$ es el individuo $i$-ésimo. Cada individuo tiene una probabilidad de ser seleccionado proporcional a su mérito. Tiene el inconveniente de que las probabilidades pueden distribuirse de forma poco equitativa (uniforme) produciendo una convergencia prematura del algoritmo. Otro inconveniente es que esta formulación no sirve cuando el mérito pueda ser un número tanto positivo como negativo.

Otro método es la selección basada en rango (ranking selection). En este caso primero se ordenan los individuos según su mérito y después se asignan probabilidades de manera proporcional en función del rango de los individuos según (10). De esta manera se produce una repartición más uniforme de la probabilidad de selección.

$$
\operatorname{Prob}^{\text {rango }}\left(P^{i}\right)=\frac{\operatorname{rango}\left(f\left(P^{i}\right)\right)}{\lambda(\lambda+1) / 2}
$$

Donde $\lambda$ es el rango (igual al tamaño de la población).

Una vez calculadas las probabilidades se suele utilizar el denominado muestreo universal estocástico (o método de la ruleta) para seleccionar individuos.

\subsubsection{Operadores de cruce}

El operador de cruce desempeña un papel fundamental en el algoritmo genético puesto que afecta directamente al nivel de diversidad de la población, y por tanto a su capacidad de explorar el espacio de parámetros.

Como se ha comentado, la formulación original de los algoritmos genéticos utiliza una codificación binaria del genoma. El proceso de cruce en un algoritmo genético de este tipo consiste en intercambiar paquetes de genes entre dos individuos. En la figura 7 se muestra un ejemplo de operador de cruce de un punto de corte. El punto de corte se elige de forma aleatoria en cada operación.

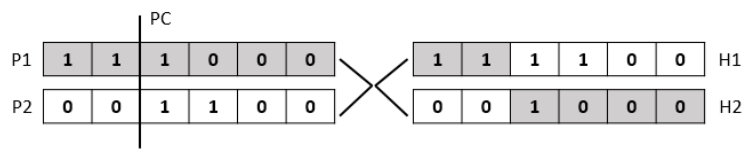

Figura 7: Ejemplo de operador de cruce binario.

Es posible resolver problemas de variable real usando algoritmos de codificación binaria, simplemente codificando las variables reales como variables binarias, pero se ha comprobado que se obtienen mejores resultados usando directamente codificación real en problemas de optimización con restricciones [7].

En un algoritmo genético de codificación real es posible aplicar el mismo método para la operación de mezcla que en un algoritmo de codificación binaria, pero hay que notar que no producirá los mismos resultados. Este tipo de cruce se denomina mezcla simple (Simple crossover). Sin embargo, se han desarrollado operadores de cruce que tratan de imitar (y mejorar) el poder de búsqueda de la operación de cruce binario. El poder de búsqueda se define como una medida de la capacidad de un operador de cruce para crear un nuevo individuo en el espacio de búsqueda. Uno de estos operadores de cruce se denomina SBX (Simulated Binary Crossover) [3] y se fundamenta en utilizar una distribución de 
probabilidad similar a la que obtiene el operador de cruce binario de un punto de corte (distribución de probabilidad polinomial). Este operador tiene el problema de que los dos hijos generados a partir de los dos padres no tienen ninguna probabilidad de aparecer en ciertas regiones del espacio [1]. Además los hijos tienen tendencia a alinearse en las direcciones de los ejes coordenados.

Existen otros tipos de cruce que tratan de evitar estos efectos indeseables del cruce SBX: BLX- $\alpha$, FR-d, PNX- $\eta$, basados en distribuciones de probabilidad uniforme, triangular y normal respectivamente [5].

En este artículo se ha utilizado el operador BLX- $\alpha$ [4] principalmente porque su formulación permite incorporar restricciones de manera natural. Además este operador tiene más capacidad de exploración del espacio de parámetros que otros operadores.

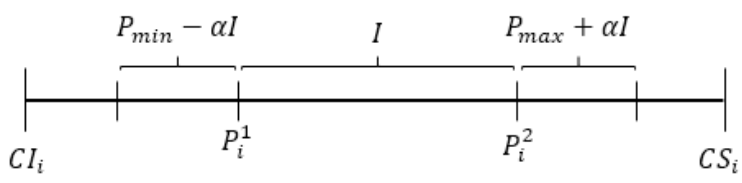

Figura 8: Operador de cruce de variable real BLX- $\alpha$. $\left(C I_{i}, C S_{i}\right)$ son las restricciones inferior y superior asociadas al parámetro o gen $i$ - ésimo.

Sean $P^{1}=\left(p_{1}^{1}, \ldots, p_{n}^{1}\right) \quad$ y $P^{2}=\left(p_{1}^{2}, \ldots, p_{n}^{2}\right) \quad$ los genomas asociados a dos padres que se cruzan. El operador BLX- $\alpha$ genera dos descendientes $H^{k}=$ $\left(h_{1}^{k}, \ldots, h_{i}^{k}, \ldots, h_{n}^{k}\right), k=1,2$, donde $h_{i}^{k}$ es un número aleatorio (uniforme) perteneciente al intervalo $\left[P_{\min }-\alpha I, P_{\max }+\alpha I\right]$, donde $P_{\text {min }}=\min \left\{P_{i}^{1}, P_{i}^{2}\right\} \mathrm{y}$ $P_{\text {max }}=\max \left\{P_{i}^{1}, P_{i}^{2}\right\}$. La figura 8 muestra gráficamente el funcionamiento del operador.

\subsubsection{Pseudo-código del algoritmo utilizado}

*Inicio AG

Generar aleatoriamente una población inicial en rango [CI,CS] MIENTRAS NO fin HACER:

*Producir una nueva generación

Calcular la FObj de cada individuo

Calcular probabilidad de selección de cada individuo (probabilidad proporcional al rango)

*Cruce

DESDE $\mathrm{i}=1$ HASTA $\mathrm{i}=\mathrm{N} / 2$ HACER:

Obtener 2 números aleatorios $[0,1]$

Seleccionar dos individuos

Cruzar los individuos con operador BLX- $\alpha$

Insertar los 2 descendientes en la nueva generación

*Mutación

Seleccionar aleatoriamente un porcentaje de individuos de la nueva generación y alterar aleatoriamente uno de los genes de cada individuo en el rango [CI,CS].

*Elitismo

Insertar al mejor individuo de la generación anterior en la nueva generación

SI numeroIteracion $=$ numeroMaxIteraciones

fin $=$ verdadero
Para comprobar que el algoritmo propuesto funciona se han hecho pruebas calculando el mínimo de la función Rastrigin [10]. Esta función tiene un mínimo global en la coordenada $(0,0)$ y muchos mínimos locales. Como el AG diseñado maximiza la función objetivo, se ha hecho uso de (8).

En las figuras 9 y 10 puede observarse cómo el algoritmo evoluciona desde una población inicial aleatoria (50 individuos) a una población final donde la mayoría de los individuos se agrupan en la vecindad del mínimo global. El factor de mutaciones elegido es 0.1 .

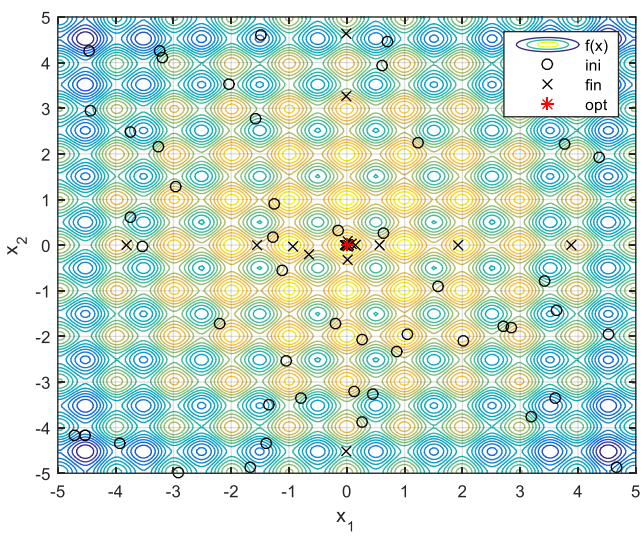

Figura 9: Poblaciones inicial y final del $\mathrm{AG}$ en el cálculo del mínimo de la función Rastrigin.

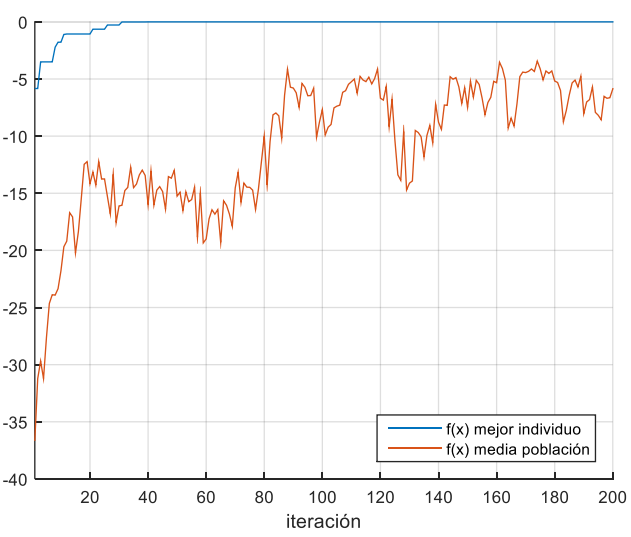

Figura 10: Evolución del valor de la función objetivo de la mejor solución y del valor medio de la función objetivo de la población con las iteraciones.

\section{RESULTADOS EXPERIMENTALES}

Para ajustar los parámetros se ha utilizado el ensayo mostrado en la figura 11, realizado el día 2 de Junio de 2016 a partir de las 11:01:47 horas. Como se observa, el ensayo se caracteriza por escalones en el caudal, 
perturbaciones en la temperatura de entrada a colectores y perturbaciones en la radiación solar producidas por nubes.

El algoritmo genético explicado en el apartado 4.1.3 se ha configurado con una población de 50 individuos, un número máximo de iteraciones igual a 60 y un porcentaje de mutaciones del $10 \%$. El valor elegido para el parámetro del operador de cruce es $\alpha=0.5$. El espacio de parámetros se ha restringido a los siguientes rangos: $C_{T} \in[2000,10000], F^{\prime} \in[0,1]$, $U_{L} \in[0,50]$.

Los valores resultantes de los parámetros de cada campo de colectores $(\mathrm{CC})$ se muestran en la tabla 3 .

La figura 15 muestra la evolución del mejor individuo, es decir, la mejor solución, con las iteraciones del algoritmo.

Para los campos CC2 y CC3 se han obtenido parámetros muy semejantes, mientras que para los campos $\mathrm{CC} 1$ y $\mathrm{CC} 4$ los parámetros se desvían respecto de los anteriores. Esta discrepancia de parámetros entre campos de colectores puede en parte explicarse por el estado envejecido de los paneles. Algunos paneles están en peores condiciones que otros (el absorbente ha perdido el color negro en algunas zonas) y algunos campos tienen más paneles en mal estado. Además los paneles no estaban limpios en el momento del ensayo, lo cual afecta directamente a la eficiencia.

Tabla 3: Resultado del ajuste de parámetros con AG.

\begin{tabular}{|l|c|c|c|c|c|}
\hline $\mathbf{C C}$ & $\boldsymbol{C}_{\boldsymbol{T}}$ & $\boldsymbol{F}^{\prime}$ & $\boldsymbol{U}_{\boldsymbol{L}}$ & $\boldsymbol{I S E}$ & $\boldsymbol{I S E} / \boldsymbol{T}$ \\
\hline 1 & 3644.67 & 0.297 & 7.23 & 1804. & 0.187 \\
2 & 5848.14 & 0.423 & 8.53 & 1046. & 0.108 \\
3 & 5523.34 & 0.406 & 8.15 & 1798. & 0.186 \\
4 & 7065.24 & 0.349 & 8.21 & 1461. & 0.151 \\
\hline
\end{tabular}

Las figuras 11 y 12 muestran los resultados del modelo ajustado por el AG para los campos de colectores CC1 y $\mathrm{CC} 2$, que son los de mayor y menor índice ISE respectivamente. Puede observarse que los dos casos el resultado del modelo ajustado, $T S C_{\text {sim }}$, se desvía poco de los valores medidos, $T S C_{\text {real }}$.

Las figuras 13 y 14 muestran el resultado de una simulación de los campos $\mathrm{CC} 1$ y $\mathrm{CC} 2$ con los parámetros de la tabla 3 y las trayectorias de un ensayo de validación realizado el día 6 de Junio de 2016 a partir de las 10:51:15 horas. Puede observarse cómo la temperatura de salida de colectores simulada presenta un offset importante respecto de la temperatura de salida de colectores medida para el campo de colectores CC1. Sin embargo, el resultado obtenido para el campo CC2 no presenta dicho offset. Tampoco lo presentan los campos CC3 y CC4.
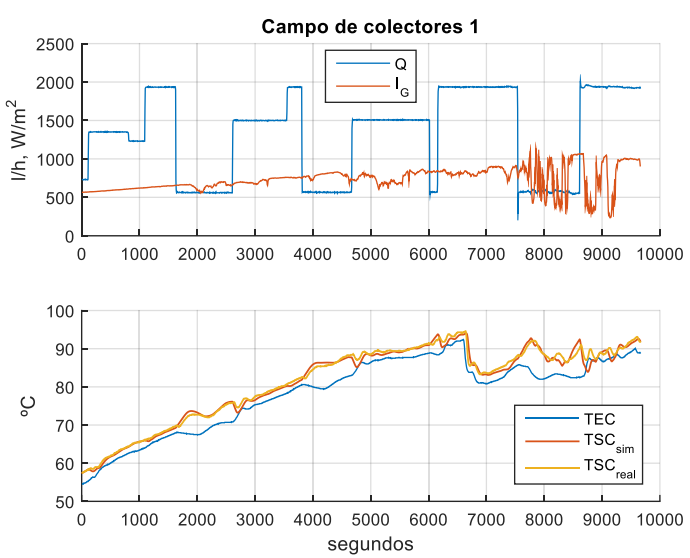

Figura 11: Resultado de la simulación del campo de colectores $\mathrm{CC} 1$.
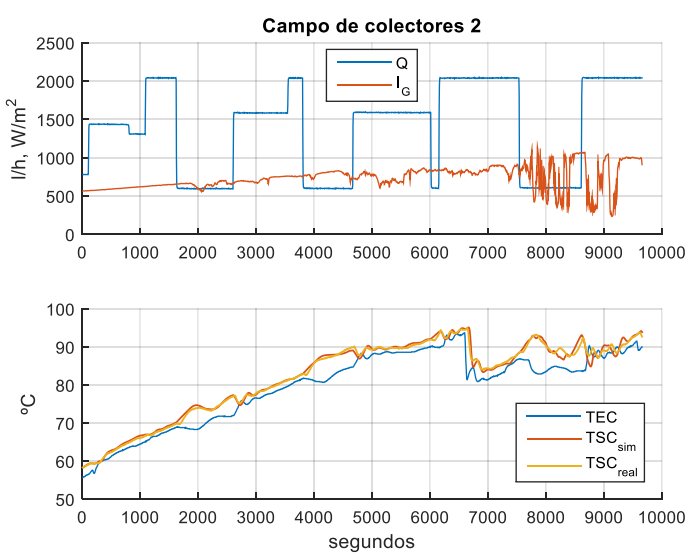

Figura 12: Resultado de la simulación del campo de colectores $\mathrm{CC} 2$.
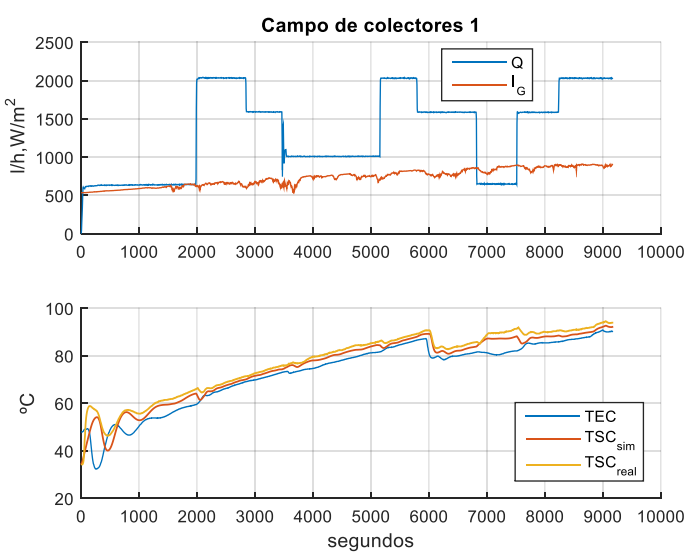

Figura 13: Resultado de la simulación del campo de colectores $\mathrm{CC} 1$ 

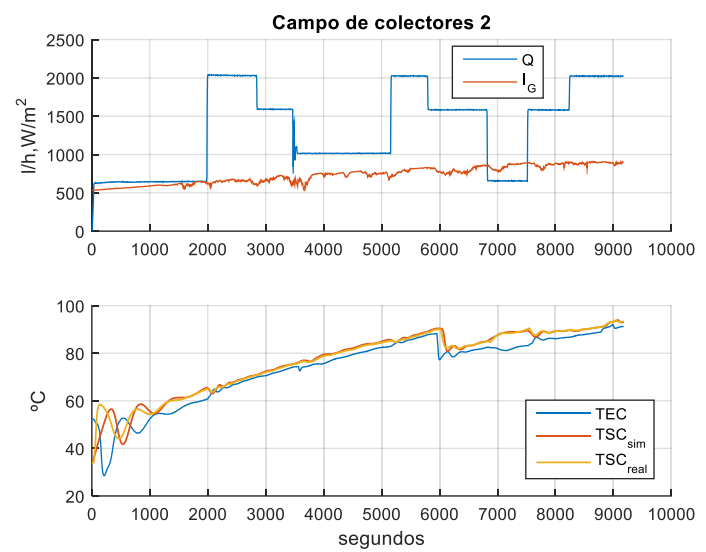

Figura 14: Resultado de la simulación del campo de colectores $\mathrm{CC} 2$.

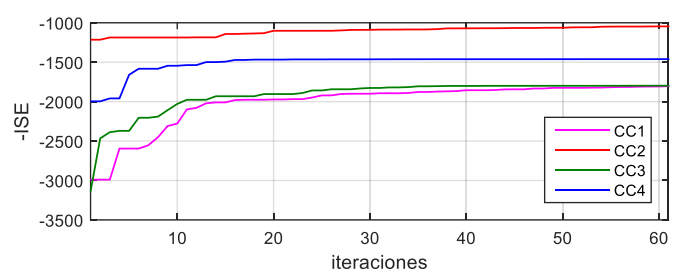

Figura 15: Evolución del valor de la función objetivo con las iteraciones del AG.

\section{CONCLUSIONES Y TRABAJOS FUTUROS}

En el presente artículo se estiman los parámetros de un modelo de colectores solares planos mediante un algoritmo genético de codificación real muy básico a partir de datos experimentales, obteniendo unos resultados aceptables. En el futuro, estos resultados se utilizarán tanto para el ajuste de controladores como para la detección y diagnosis de fallos en la planta. Se ha observado además cómo las condiciones ambientales (temperatura ambiente, velocidad $\mathrm{y}$ dirección del viento, radiación, etc.) afectan a los parámetros estimados, por lo que queda abierta una línea de investigación para intentar establecer una correlación entre las condiciones ambientales instantáneas y los parámetros asociados a las pérdidas globales al ambiente y la eficiencia.

\section{Agradecimientos}

Los autores quieren expresar su agradecimiento al Ministerio de Ciencia e Innovación por la financiación de este trabajo a través del proyecto DPI2013-44135$\mathrm{R}$.

\section{Referencias}

[1] Ballester, P. J., \& Carter, J. N. (2004, June). An effective real-parameter genetic algorithm with parent centric normal crossover for multimodal optimisation. In Genetic and Evolutionary Computation-GECCO 2004 (pp. 901-913).

[2] Cruz-Peragon, F., Palomar, J. M., Casanova, P. J., Dorado, M. P., \& Manzano-Agugliaro, F. (2012). Characterization of solar flat plate collectors. Renewable and sustainable energy reviews, 16(3), 1709-1720.

[3] Deb, K., \& Agrawal, R. B. (1994). Simulated binary crossover for continuous search space. Complex Systems, 9(3), 1-15.

[4] Herrera, F., Lozano, M., Pérez, E., Sánchez, A. M., \& Villar, P. (2002). Multiple crossover per couple with selection of the two best offspring: an experimental study with the BLX- $\alpha$ crossover operator for real-coded genetic algorithms. In Advances in Artificial IntelligenceIBERAMIA 2002 (pp. 392-401).

[5] Herrera, F., Lozano, M., \& Sánchez, A. M. (2007). Algoritmos Genéticos con Codificación Real: Operadores de Cruce Híbridos Basados en Entornos con Múltiples Descendientes. In Actas del Congreso MAEB (Vol. 7, pp. 827-834).

[6] Herrera, F., Lozano, M., \& Verdegay, J. L. (1998). Tackling real-coded genetic algorithms: Operators and tools for behavioural analysis. Artificial intelligence review, 12(4), 265-319.

[7] Iruthayarajan, M. W., \& Baskar, S. (2009). Evolutionary algorithms based design of multivariable PID controller. Expert Systems with applications, 36(5), 9159-9167.

[8] Klein, S. A., Duffie, J. A., \& Beckman, W. A. (1974). Transient considerations of flat-plate solar collectors. Journal of Engineering for Power, 96(2), 109-113.

[9] López, S. H., Perez, S. L., del Hoyo Arce, I., \& Dávila, I. M. (2015, September). Dynamic Modelling of a Flat-Plate Solar Collector for Control Purposes. In Proceedings of the 11th International Modelica Conference, Versailles, France, 2015 (No. 118, pp. 419-426).

[10] Rastrigin, L. A. (1974). Systems of extremal control. Zinatne, Riga.

[11] Reiter, C. N., Trinkl, C., Zörner, W., \& Hanby, V. I. (2015). A Dynamic Multinode Model for Component-Oriented Thermal Analysis of FlatPlate Solar Collectors. Journal of Solar Energy, 2015. 